advanced, previously untreated cervical cancer will be randomized 1:1 to receive either 5 cycles of pembrolizumab $200 \mathrm{mg}$ Q3W plus CCRT followed by 15 cycles of pembrolizumab $400 \mathrm{mg}$ Q6W or 5 cycles of placebo Q3W plus CCRT followed by 15 cycles of placebo Q6W. CCRT includes 5 cycles (optional 6th dose) of cisplatin $40 \mathrm{mg} / \mathrm{m}^{2}$ Q1W plus EBRT followed by brachytherapy. Randomization is stratified by planned EBRT type (intensity-modulated radiotherapy [IMRT] or volumetric-modulated arc therapy [VMAT] vs non-IMRT or non-VMAT), screening cancer stage (IB2-IIB vs III-IVA), and planned total radiotherapy dose. Treatment will continue for 20 cycles or disease progression, unacceptable toxicity, or withdrawal. Primary endpoints are PFS per RECIST v1.1 by investigator and OS. Secondary endpoints include PFS by BICR, PFS at 2 years, OS at 3 years, CR at 12 weeks, ORR, OS and PFS by PD-L1 status, QoL, and safety. Enrollment began May 2020 and is planned for 193 sites in 30 countries. Results Not applicable

Conclusions Not applicable

\section{EPV037/\#157 INCIDENCE OF CERVICAL CANCER AND THE HPV VACCINE IN THE UNITED STATES: ARE WE SEEING RESULTS OF VACCINATION EFFORTS?}

${ }^{1} \mathrm{~A}$ Francoeur, ${ }^{2} \mathrm{C}-\mathrm{I}$ Liao, ${ }^{1} \mathrm{D}$ Wong, ${ }^{3} \mathrm{~A}$ Mann, ${ }^{4} \mathrm{MA}$ Caesar, ${ }^{5} \mathrm{~A}$ Chan, ${ }^{6} \mathrm{~B}$ Monk, ${ }^{7} \mathrm{D}$ Kapp, ${ }^{8} \mathrm{~J}$ Chan. 'University of California Los Angeles, Obstetrics and Gynecology, Los Angeles, USA; ${ }^{2}$ Kaohsiung Veterans General Hospital, Obstetrics and Gynecology, Kaohsiung City, Taiwan; ${ }^{3} \mathrm{Pal}$ Alto Medical Foundation, Research Institute, Palo Alto, USA; ${ }^{4}$ California Pacific Medical Center, Research Institute, San Francisco, USA; ${ }^{5}$ Palo Alto Medical Foundation Research Intitute, Obstetrics and Gynecology, Palo Alto, USA; ${ }^{6}$ Arizona Oncology, Gynecologic Oncology, Obstetrics and Gynecology, Phoenix, USA; ${ }^{7}$ Stanford University School of Medicine, Department of Radiation Oncology, Stanford, USA; ${ }^{8}$ California Pacific Medical Center, Obstetrics and Gynecology, San Francisco, USA

\subsection{6/ijgc-2021-IGCS.105}

Objectives To determine the incidence and trends of cervical cancer in the United States in relation to the HPV vaccine.

Methods Data were obtained from the U.S. Cancer Statistics program from 2001-2017. SEER*Stat 8.3.8 and Joinpoint regression program 4.8.0.1 were used to calculate incidence trends.

Results Over the last 17 years, cervical cancer incidence is decreasing at an average annual percent change (AAPC) of $1.03 \%(\mathrm{p}<0.001)$. We performed a subset analysis of women who were 9-13 years old in 2006 when the HPV vaccine was approved, now 20-24 years old in 2017. In the pre-vaccine era (2001-2011), the incidence of cancer decreased $2.3 \%$ annually $(p=0.038)$. of note, after the introduction of the vaccine $(2011-2017)$, it decreased at $9.6 \%$ per year $(p=0.002)$. In the pre-vaccine era (2001-2012), the incidence of new diagnoses of squamous cell carcinoma observed a decrease of $3.1 \%$ annually $(\mathrm{p}=0.004)$. However, in the post-vaccine era (2012-2017), there was an $11.8 \%$ decline in new cases per year $(p=0.007)$. Although there is a decrease in older age groups, there is no difference in the trends pre and post vaccine era, particularly in the age groups who were not eligible for vaccination at that time.

Conclusions In our population analysis, our data suggest that the HPV vaccination may have decreased in incidence of cervical cancer in the younger cohort after its approval.
EPV038/\#163 NEUROTROPHIC TYROSINE KINASE RECEPTOR-1 (NTRK-1) REARRANGED CERVICAL SARCOMA WITH FIBROSARCOMA LIKE MORPHOLOGY PRESENTING IN A 13-YEAR-OLD MANAGED WITH A NEO-ADJUVANT TRK-INHIBITOR AND SURGICAL EXCISION

${ }^{1} \mathrm{E}$ Goulding*, ${ }^{2} \mathrm{P}$ Morreau, ${ }^{3} \mathrm{M}$ De Silva, ${ }^{4} \mathrm{M}$ Watson, ${ }^{5} \mathrm{~B}$ Leung, ${ }^{6} \mathrm{C}$ Van Vliet, ${ }^{1} \mathrm{~L}$ Eva. ${ }^{1}$ National Women's Health, Gynaecologic Oncology, Auckland, New Zealand; '2Starship Children's Hospital, Paediatric Surgery, Auckland, New Zealand; '3tarship Children's Hospital, Medical Oncology, Auckland, New Zealand; ${ }^{4}$ Auckland City Hospital, Anatomical Pathology, Auckland, New Zealand; ${ }^{5}$ Starship Children's Hospital, Radiology, Auckland, New Zealand; ${ }^{6}$ QEIl Medical Centre, Anatomical Pathology, Perth, Australia

\subsection{6/ijgc-2021-IGCS. 106}

Objectives Fibrosarcoma like tumours of the uterine cervix affecting premenopausal women with neurotrophic tyrosine kinase receptor (NTRK) gene rearrangements have recently been described in the literature. They are rare tumours and to our knowledge there are only 18 cases reported, none of which has occurred in the paediatric population. We describe the first case of a paediatric patient with a NTRK fusion positive fibrosarcoma-like tumour of the uterine cervix who was successfully managed with neo-adjuvant entrectinib and subsequently went on to have conservative, fertility sparing surgery. Methods This case report reviews the case of a 13-year-old patient who presented with a $9 \mathrm{~cm}$ NTRK-1 rearranged cervical sarcoma with fibrosarcoma like morphology. At presentation the lesion filled her vagina and pelvis and any attempt at surgical removal would have been morbid and led to loss of fertility.

Results Based upon evidence that has shown good tolerability and responses of paediatric solid tumours with NTRK gene fusions to NTRK inhibitors, both in the neoadjuvant and upfront setting, this patient was managed with neo-adjuvant entrectinib. Following a dramatic reduction in tumour size confirmed by imaging, she underwent conservative fertility sparing surgery with final histopathology showing no residual disease.

Conclusions This case highlights the importance of the investigation of NTRK fusions in fibrosarcoma like tumours of the uterine cervix, as this may open up treatment options for patients and avoids potentially morbid extensive surgery, which may impair fertility.

\section{EPV039/\#175 RELATIVE IMPORTANCE OF INDIVIDUAL INSURANCE STATUS AND HOSPITAL PAYER MIX ON SURVIVAL FOR WOMEN WITH CERVICAL CANCER}

${ }^{1} \mathrm{C}$ Cherston*, ${ }^{1} Y$ Huang, ${ }^{1} \mathrm{~A}$ Melamed, ${ }^{2} \mathrm{~V}$ Prabhu, ${ }^{2} \mathrm{Y}$ Li, ${ }^{1} \mathrm{~J}$ Wright. ${ }^{1}$ Columbia University, Gynecologic Oncology, New York, USA; ${ }^{2}$ Merck and Co., Inc., Outcomes Research, Kenilworth, USA

\subsection{6/ijgc-2021-IGCS. 107}

Objectives Safety-net hospitals (SNH) are important sites of care especially for vulnerable groups (e.g., uninsured/Medicaid). We examined the relative contributions of individual insurance status and hospital payer mix on quality of care and survival for women with cervical cancer. 
Abstract EPV039/\#175 Table 1 Individual insurance status, Hospital payer mix and associated mortality among cervical cancer patients

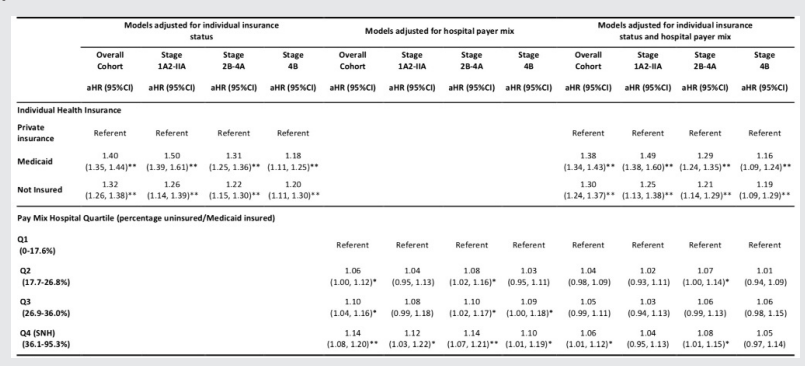

${ }^{*} \mathrm{P}<0.05,{ }^{*} \mathrm{P}<0.001$.

Marginal cox proportional hazards models adjusted for hospital clustering, patient's age, race, year of diagnosis, zip code median house income quartile, charlson comorbidity score, cancer substage, histology, grade and tumor size. Values reported as hazard ratios with $95 \%$ confidence interval.

Change of AIC (Akaike information criterion) in multivariable model omitting individual insurance $=731$; change of AIC in multivariable model omitting individual insurance $=10$.

Methods We used the National Cancer Database to identify cervical cancer patients diagnosed 2004-2017. Patients were classified by insurance status (Medicaid/uninsured vs. private) and hospitals were grouped into quartiles based on the proportion of uninsured/Medicaid patients (payer mix) (top quartile defined as SNHs). Quality-of-care was assessed by adherence to evidence-based metrics and survival by proportional hazards models. Individual contributions of insurance status and hospital payer mix on quality-of-care and survival were assessed.

Results A total of 124,339 patients including 11,338 uninsured (9.1\%) and 27,281 Medicaid (21.9\%) recipients treated at 1156 hospitals were identified. Quality-of-care was not significantly different across hospital quartiles. Adjusting for clinical/ demographic characteristics and hospital payer mix, treatment at a $\mathrm{SNH}$ was associated with a $14 \%$ higher mortality $(\mathrm{HR}=1.14 ; 95 \% \mathrm{CL}, 1.08-1.20)$ than Q1 hospitals. Adjusting for individual insurance, uninsured women had 32\% increased mortality $(\mathrm{HR}=1.32 ; 95 \% \mathrm{CI}, 1.26-1.38)$ and Medicaid recipients $40 \%$ increased $(\mathrm{HR}=1.40 ; 95 \% \mathrm{CI}, 1.35-1.44)$ compared to privately insured subjects. Adjusting for both payer mix and insurance status, only individual insurance retained a significant impact on mortality (table 1).

Conclusions Individual insurance status (having Medicaid or no insurance) may be a more important predictor of survival than site of care and hospital payer mix for women with cervical cancer.

\section{EPV040/\#185 THE PROGNOSTIC VALUE OF THE NUMBER OF POSITIVE LYMPH NODES AND THE LYMPH NODE RATIO IN EARLY STAGE CERVICAL CANCER}

${ }^{1} \mathrm{E}$ Olthof*, ${ }^{2} \mathrm{C}$ Mom, ${ }^{1} \mathrm{H}$ Wenzel, ${ }^{2} \mathrm{~J}$ Van Der Velden, ${ }^{1} \mathrm{M}$ Van Der Aa. ${ }^{1}$ Netherlands Comprehensive Cancer Organization, Research and Development, Utrecht, Netherlands; ${ }^{2}$ Amsterdam University Medical Centre, Gynaecological Oncology, Amsterdam, Netherlands

\subsection{6/ijgc-2021-IGCS.108}

Objectives Lymph node metastases are now incorporated into the 2018 International Federation of Gynecology and Obstetrics (FIGO) staging system for cervical cancer. However, the
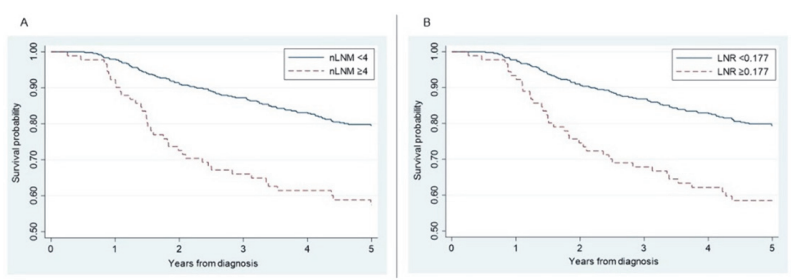

Abstract EPV040/\#185 Figure 1 Kaplan-Meier curves, 5-year overall survival categorizes by (A) nLNM risk-groups and (B) LNR risk-groups.

number of positive lymph nodes (nLNM) or the lymph node ratio (LNR) might provide a better prediction of survival. The aim of this study is to establish the impact of nLNM and LNR on survival in early-stage cervical cancer patients after surgery.

Methods In this population-based study, we selected all women diagnosed between 1995-2020 with FIGO 2009 stage IA2IIA1 cervical cancer and nodal metastases after radical hysterectomy and pelvic lymphadenectomy from the Netherlands Cancer Registry. Optimal cut-offs for prognostic stratification by $\mathrm{nLNM}$ and LNR were calculated to categorize patients in low- or high-risk groups. Kaplan-Meier overall survival analysis and flexible parametric relative survival analysis were used to determine the impact of nLNM and LNR on survival. Missing data were imputed.

Results Of 593 patients, 500 and 501 (84\%) were categorized in the low-risk and 93 and $92(16 \%)$ in the high-risk groups for nLNM $(\geq 4)$ and LNR $(\geq 0.177)$, respectively. Both highrisk groups had a worse 5 -year overall survival $(p<0.001)$ and were, together with non-squamous histology, independent risk factors for relative survival, with excess hazard ratios of 2.4 (95\% CI 1.6-3.5) for nLNM and 2.5 (95\% CI 1.7-3.8) for LNR.

Conclusions Presenting a patient's nodal status postoperatively by the number of positive nodes, or by its ratio, can support further risk stratification regarding survival in case of nodepositive early-stage cervical cancer.

\section{EPV041/\#196 HYSTERECTOMY AFTER CHEMORADIOTHERAPY FOR LOCALLY ADVANCED CERVICAL CANCER - EVALUATION OF PROGNOSTIC FACTORS AND SURVIVAL}

T Almeida De Sousa*, D Falcao Filho, M Amorim Nascimento, S Medeiros, R Oliveira Macedo, P Zanvettor. Aristides Maltez Hospital, Gynecologic Oncology, Salvador, Brazil

\subsection{6/ijgc-2021-IGCS.109}

Objectives Evaluate survival and prognostic factors of surgery after chemoradiotherapy (CRT) for locally advanced cervical cancer (LACC).

Methods A retrospective study was performed comparing patients who had undergone surgery following primary CRT for LACC to a control group treated only with CRT.

Results 176 patients fulfilled the inclusion criteria. Residual disease (RD) was found in $48(55,2 \%)$ patients submitted to surgery, $32(66,7 \%)$ had adenocarcinoma $(p=0,054)$. The main prognostic factor related to $\mathrm{RD}$ in a multivariate analysis was adenocarcinoma histologic type $(\mathrm{p}=0,005, \mathrm{HR}=5,54$ $(1,69-18,12))$. Patients with RD presented higher recurrence 